We hope to follow the success of the mhGAP programme and implement mhGAP-ID in other countries through forums such as the Royal College of Psychiatrists International Links working group on intellectual disability. Combined mhGAP and mhGAP-ID training would allow efficient use of sometimes scarce resources and opportunities.

\section{References}

Abdulmalik, J., Kola, L., Fadahunsi, W., et al (2013) Country contextualization of the Mental Health Gap Action Programme Intervention Guide: a case study from Nigeria. PLoS Med, 10, e1001501. doi:10.1371/journal.pmed.1001501.

Beiser, M., Simich, L. \& Pandalangat, N. (2003) Community in distress: mental health needs and help seeking in the Tamil community in Toronto. International Migration, 41, 233-245.

Fernando, S. \& Weerackody, C. (2009) Challenges in developing community mental health services in Sri Lanka. Journal of Health Management, 11, 195-208.

Jenkins, R., Mendis, J., Cooray, S., et al (2012) Integration of mental health into primary care in Sri Lanka. Mental Health in Family Medicine, 9, 15-24.
Khon, R., Saxena, D., Levav, I., et al (2004) The treatment gap in mental health care. Bulletin of the World Health Organization, 82, 858-864.

Royal College of Psychiatrists (2011) Minority Ethnic Communities and Specialist Learning Disability Services. RCPsych.

Samarasekara, N., Davies, M. \& Siribaddana, S. (2012) The stigma of mental illness in Sri Lanka: the perspectives of community mental health workers. Stigma Research and Action, 2(2). doi:10.5463/sra. v1i3.48

Saxena, S. (2011) News from the WHO Mental Health Gap Programme (mhGAP). World Health Organization mhGAP Newsletter, 11 June.

WHO (2007) Atlas: Global Resources for Persons with Intellectual Disabilities. World Health Organization.

WHO (2008) mhGAP Mental Health Gap Action Programme: Scaling Up Care for Mental, Neurological, and Substance Use Disorder. World Health Organization.

WHO (2009) ECOSOC Meeting: Mental Health, Poverty and Development (Discussion Paper). World Health Organization.

WHO (2011) Human Resources for Mental Health: Workforce Shortages in Low- and Middle-Income Countries (Human Resources for Health Observer No. 8). World Health Organization.
RESEARCH PAPER

\section{Acceptability and challenges of} implementing the NICE guidelines for schizophrenia in Lagos, Nigeria

\author{
Adeniran Okewole, ${ }^{1}$ Mobolaji Dada, ${ }^{2}$ Adefemi Adeoye, ${ }^{3}$ Kehinde Lawal ${ }^{4}$ and \\ Taiwo Oduguwa ${ }^{5}$
}

${ }^{1}$ Consultant Psychiatrist Neuropsychiatric Hospital, Aro, Abeokuta, Nigeria, emai niranokewole@gmail.com

${ }^{2}$ Consultant Psychiatrist, Ekiti State University Teaching Hospital, Ekiti, Nigeria

${ }^{3}$ Consultant Psychiatrist, Federal Neuropsychiatric Hospital, Yaba Lagos, Nigeria

${ }^{4}$ Senior Registrar, Centre for Child and Adolescent Mental Health,

University College Hospital,

Ibadan, Nigeria

${ }^{5}$ Consultant Psychiatrist, Federal Neuropsychiatric Hospital, Yaba, Lagos, Nigeria
This study aimed to examine the suitability and challenges of implementing in a Nigerian tertiary mental health facility the guidelines for the management of schizophrenia produced by the UK National Institute for Health and Care Excellence (NICE). The study was based on a group discussion at the Psychiatric Hospital, Yaba, Lagos. A panel of ten individuals (consultants, senior registrars, senior psychologists and senior social workers and nurses) discussed the guidelines, having been given ample time to study the document. Five patients were also interviewed. Some of the recommendations of the NICE guidelines are already being practised in the hospital to various extents. Full implementation would be hampered by a shortage of human resources and financial constraints. The guidelines need to factor in sociocultural differences. The NICE guidelines with modifications are suitable for use in a Nigerian setting.

Clinical guidelines aim to assist health workers and service users in making effective decisions about the management of specific clinical conditions. They strive to implement available evidence and bridge the gap between research and practice (Gray, 2005). Ample evidence exists about the usefulness of guidelines. Grimshaw \& Russell (1993) did a systematic review of 59 published evaluations of clinical guidelines, of which 55 detected significant improvement in the process of care after the guidelines were introduced.

The guidelines for schizophrenia produced by the National Institute for Health and Care Excellence (NICE; formerly the National Institute for Clinical Excellence) were developed through collaboration between professionals, service users and carers (NICE, 2002, 2009). They address major treatments and services for people with schizophrenia, are evidence-based and each recommendation is graded according to level of evidence (Gray, 2005). The guidelines divide the treatment and management of schizophrenia into three phases: initiation of treatment of the first episode; acute phase; and promotion of recovery. They recommend collaboration with service users and carers in each phase. The use of care notes and care plans is also encouraged. Crisis resolution, home-treatment teams, early-intervention teams, community mental health teams and acute day hospitals are recommended, with in-patient treatment if necessary. The treatment package 
also includes comprehensive needs assessment, cognitive-behavioural therapy (CBT) and family interventions.

With respect to medication, atypical antipsychotics are preferred, although the revised version calls for a balance of cost and benefit. Depots should be considered for non-adherent users, and clozapine should be introduced only after a trial of two different antipsychotics (including one atypical).

Rowlands (2004) evaluated the challenges of implementing the NICE guidelines at the individual and organisational level. He identified the key issues in implementation as dissemination, ownership, barriers to change and sustainability. The resource implications, even in an affluent society, can be enormous. In low-income countries like Nigeria, home-grown guidelines are scarce, with the result that guidelines either do not exist or are imported. This study examined the acceptability and challenges of implementing practice guidelines, using the specific example of the NICE guidelines for schizophrenia.

\section{Method}

The study was conducted at the Psychiatric Hospital, Yaba, Lagos, Nigeria. In a group discussion with dual moderators, ten members of staff (consultants, senior registrars, senior psychologists and senior social workers and nurses) evaluated the NICE guidelines for schizophrenia, after studying the document (copies of which were made available to each discussant). The discussion session was recorded on tape and subsequently transcribed. Questions for discussion were selected by the group to cover the various aspects of the guidelines. Ethical approval was obtained from the hospital's ethics and research committee. Analysis of the transcribed text was done using NVIVO for qualitative research (version 8).

After the discussion, five patients on admission with a diagnosis of schizophrenia were interviewed to obtain their perspective on various aspects of the guidelines.

\section{Results}

The discussants were unanimous about the need for practice guidelines in the hospital. These, they pointed out, would help to harmonise and standardise practice. A collaborative approach to management, as recommended by NICE, was adjudged desirable in achieving the overall goal of quality patient care. Care notes and care plans were also deemed beneficial.

They observed that atypical antipsychotic medication had become more accessible in view of the new generic brands. For those who cannot access them, it may still be necessary to rely on conventional antipsychotics.

The major identified barrier to a communitybased approach was the shortage of human resources. Suggestions included outreach teams and staff training in community mental health services. Nurses and social workers could be engaged in doing an initial needs assessment, after which the whole team could deliver outreach and community services. Primary health centres could provide platforms for community intervention.

Cognitive-behavioural therapy and family interventions targeted at relapse prevention, reduction of symptoms, improvement in insight and promotion of medication adherence were considered vital. A barrier to this may again be the shortage of trained staff. Statements which summarised the opinion of the discussants include 'We should look at the illness itself and also the manpower' and 'We must tailor what comes from abroad to suit our purpose'.

The discussants unanimously voted for adoption of the NICE guidelines by the hospital. It was pointed out, however, that modifications would need to be made. Other guidelines may also need to be consulted in fashioning a document for use in the hospital.

While some interviewed patients felt the choice of atypical antipsychotics could not be so easily made due to cost, a couple stated that they were comfortable with whatever the doctor prescribed. One of the patients responded that the guidelines were 'a good way of taking care of us'.

\section{Discussion}

This study addresses the feasibility of implementing management guidelines in a resource-constrained setting like Nigeria, using the NICE guidelines for schizophrenia as an example. The cost implications of managing schizophrenia in a low-income country like Nigeria can be quite enormous (Suleiman et al, 1997), bearing in mind that a large proportion of the populace live below the poverty line. The inadequacy or outright unavailability of social support systems such as welfare and disability benefits and unemployment benefits means that many patients cannot access proper healthcare. While some early studies highlighted the possibility of good outcomes in Nigerian patients with schizophrenia compared with those in highincome countries, others have disputed this claim (Gureje \& Bamidele, 1994).

The human resource challenge of managing schizophrenia in line with guidelines such as NICE's can be enormous. Whereas community management is preferred, there are often barely enough workers to cater to the hospital in-patient population, leaving very few to attend to patients at home or in the community. Treatment modalities such as psychotherapy are often not administered or inadequately administered due to the pressure on the few sufficiently trained personnel.

The importance of the cultural milieu and the need to adapt guidelines and interventions to suit the particularities of the given culture were also highlighted. These include positive ones such as the extended family system and negative ones like a widespread attribution of mental illness to spiritual causes and the tendency to seek unorthodox care. With specific reference to the cultural adaptability of CBT, a randomised controlled trial involving 
patients with schizophrenia from ethnic minorities in the UK revealed that those who participated in a culturally adapted form of CBT for psychosis achieved significantly better results than those who received treatment as usual, with some gains maintained at follow-up (Rathod et al, 2013). High levels of satisfaction were also reported. A preliminary evaluation from Pakistan (Habib et al, 2014) also reported that culturally adapted CBT was effective in reducing symptoms of psychosis and in improving insight in in-patient settings.

Another key area of adaptability is the use of medication. In keeping with recent research, the NICE guidelines have been revised to better accommodate conventional antipsychotics. While atypical antipsychotics are the preferred option in most high-income countries, a robust body of research has examined the efficacy and side-effect profile of conventional versus atypical antipsychotics; the conclusion is that, with clozapine as a notable exception, the cost of atypical antipsychotics is often unjustified (Bruijnzeel et al, 2014).

\section{Conclusion}

The NICE guidelines are a useful template for care in Nigeria. Constraints to full implementation include human resources and cost. The guidelines need to factor in sociocultural differences. With modifications, they are suitable for use in Nigeria.

\section{References}

Bruijnzeel, D., Suryadevara, U. \& Tandon, R. (2014) Antipsychotic treatment of schizophrenia: an update. Asian Journal of Psychiatry, 11, 3-7. doi: 10.1016/j.ajp.2014.08.002.

Gray, R. (2005) The NICE guidelines for schizophrenia. Psychiatry, 4(10), 11-13.

Grimshaw, J. M. \& Russell, I. T. (1993) Effect of clinical guidelines on medical practice: a systematic review of rigorous evaluation. Lancet, 342, 1317-1322.

Gureje, O. \& Bamidele, R.(1994) Thirteen year social outcome among Nigerian outpatients with schizophrenia. Social Psychiatry and Psychiatric Epidemiology, 34, 147-151.

Habib, N., Dawood, S., Kingdon, D., et al (2014) Preliminary evaluation of culturally adapted CBT for psychosis (CA-CBTp): findings from Developing Culturally-Sensitive CBT Project (DCCP). Behavioural and Cognitive Psychotherapy, 2, 1-9.

NICE (2002) Clinical Guideline 1. Schizophrenia: Core Interventions in the Treatment and Management of Schizophrenia in Primary and Secondary Care. London: NICE.

NICE (2009) Schizophrenia: Core Interventions in the Treatment and Management of Schizophrenia in Primary and Secondary Care. NICE.

Rathod, S., Phiri, P., Harris, S., et al (2013) Cognitive behavior therapy for psychosis can be adapted for minority ethnic groups: a randomized controlled trial. Schizophrenia Research, 143, 319-326.

Rowlands, P. (2004) The NICE schizophrenia guidelines: the challenge of implementation. Advances in Psychiatric Treatment, $10,403-412$.

Suleiman, G. T. Ohaeri, J. U., Lawal, R. A et al (1997) Financia cost of treating outpatients with schizophrenia in Nigeria. British Journal of Psychiatry, 171, 364-368.

\section{RESEARCH} PAPER

\title{
Mental health research in the Arab world: an update
}

\author{
Elie G. Karam ${ }^{1}$ and Lynn A. Itani ${ }^{2}$
}

'Professor and Head, Institute for Development, Research, Advocacy and Applied Care (IDRAAC), Medical Institute for Neuropsychological Disorders (MIND), Department Disorders (MIND), Departme
of Psychiatry and Clinical Psychology, St George Hospital University Medical Center, Balamand University, Faculty of Medicine, Beirut, Lebanon, email egkaram@idraac.or

2Research Associate, Institute for Development Research Advocacy and Applied Care (IDRAAC).

Beirut, Lebanon

Acknowledgement: Mr Rami Ofeish helped with the

production of the results reported in this article.
Publications on mental health were collected using PubMed and PsychINFO for 21 Arab countries. The data were then categorised according to the first author's country of affiliation, the year of publication, the topic of research and the type of journal. In 2006-12, the Arab world published 1029 articles (an average of 147 per year). The estimated increase in yearly productivity during this period was about $25 \%$ over the 7 preceding years. When considering the research output per million population, Kuwait, Bahrain and Lebanon were the top three producers, as they had been over the preceding four decades. After adjusting for gross domestic product (GDP) per capita, the five top producers were Egypt, Jordan, Tunisia, Lebanon and Morocco. Based on child and adolescent mental health research only, the Arab world's productivity was around one-sixth that of the United States and Europe.
Mental disorders are significant contributors to the burden of disease in the Arab region (World Health Organization, 2008). Mental health research sheds light on local data such as the prevalence of disorders and the extent and modalities of treatments, which are crucial in planning national policies.

In a previous study, we identified 2213 published articles related to mental health from the Arab region over four decades (1966-2005) (Jaalouk et al, 2012). We estimated that Arab countries produce around one-sixth of the global output of mental health research, an amount comparable to Latin America and Caribbean countries (Saxena $e t$ al, 2006). That output had been growing fast: in the last decade of the study period (1996-2005), Arab countries produced eight times more publications than their average for 1966-75 and 1976-85 and double that for 1986-95. This productivity varied widely and when publications were calculated per million population, the top publishing countries 